\title{
Estudio clínico randomizado comparando agentes antisépticos en la piel en la operación cesárea (1)
}

\author{
Tuuli MG, Liu J, Stout MJ, Martin S, Cahill AG, Odibo AO, Colditz GA, Macones GA. A \\ Randomized Trial Comparing Skin Antiseptic Agents at Cesarean Delivery. N Engl J \\ Med 2016;374(7):647-55.
}

Análisis Crítico: Jorge A. Carvajal PhD.

Unidad de Medicina Materno-Fetal, División de Obstetricia y Ginecología, Facultad de Medicina, Pontificia Universidad Católica de Chile.

\section{RESUMEN (1)}

Antecedentes: La antisepsia preoperatoria de la piel tiene el potencial de disminuir el riesgo de infección del sitio quirúrgico. Sin embargo, la evidencia es limitada para orientar la elección del agente antiséptico en el parto por cesárea, que es el procedimiento de cirugía mayor más común entre las mujeres en los Estados Unidos. Métodos: En este estudio aleatorizado, controlado, en un solo centro, se evaluó si el uso de clorhexidina-alcohol para la antisepsia preoperatoria de la piel era superior al uso de yodo-alcohol para la prevención de infección del sitio quirúrgico después de un parto por cesárea. Asignamos al azar a los pacientes sometidos a parto por cesárea a preparación de la piel con clorhexidina-alcohol o yodo-alcohol. El resultado primario fue infección superficial o profunda del sitio quirúrgico dentro de los 30 días después del parto por cesárea, basados en las definiciones del Centro para el Control y la Prevención de Enfermedades Infecciosas. Resultados: Desde septiembre de 2011 hasta junio de 2015, un total de 1.147 pacientes fueron enroladas; 572 pacientes fueron asignados a clorhexidina-alcohol y 575 a yodoalcohol. En un análisis por intención de tratar, la infección del sitio quirúrgico fue diagnosticada en 23 pacientes $(4,0 \%)$ en el grupo de clorhexidinaalcohol y en $42(7,3 \%)$ en el grupo de yodo-alcohol (riesgo relativo: 0,55; IC95\% 0,34-0,90; p=0,02). La tasa de infección superficial de la herida quirúrgica fue del 3,0\% en el grupo de clorhexidina-alcohol y $4,9 \%$ en el grupo de yodo-alcohol $(p=0,10)$; la tasa de infección profunda fue de $1,0 \%$ y $2,4 \%$, respectivamente $(p=0,07)$. La frecuencia de reacciones adversas de la piel fue similar en los dos grupos. Conclusiones: El uso de clorhexidina-alcohol para la antisepsia preoperatoria de la piel resulta en un riesgo significativamente menor de infección del sitio quirúrgico después de una cesárea comparado con la infección asociada al uso de yodo-alcohol.

\section{ANÁLISIS DE LA INVESTIGACIÓN}

\section{A. Relevancia clínica de la investigación}

La operación cesárea se efectúa en Chile con frecuencia creciente; estimaciones recientes indican que cerca del $40 \%$ de los partos en Chile ocurren mediante operación cesárea, siendo esta cifra una de las mayores entre los países miembros de la OCDE (2). La operación cesárea presenta múltiples complicaciones que justifican que su uso debe ser restringido. Una de las principales complicaciones de la operación cesárea es la infección del sitio quirúrgico, la que afecta habitualmente entre $5-12 \%$ de los casos (3). Se han descrito múltiples estrategias para reducir el riesgo de infección, siendo las principales medidas de prevención el uso de una correcta técnica quirúrgica, los antibióticos preoperatorios y el aseo de la piel con antisépticos (3). Sin embargo, la evidencia no es concluyente respecto a cuál es el mejor antiséptico a utilizar en el aseo de la piel de la zona operatoria. En chile, los dos antisépticos de uso más difundido son la clorhexidina y la povidona iodada. 
Tabla I

RIESGO RELATIVO DE INFECCIÓN DE LA HERIDA OPERATORIA EN CESÁREAS. CLORHEXIDINA-ALCOHOL VS YODO-ALCOHOL

\begin{tabular}{lcccc}
\hline Infección & Clorhexidina alcohol & Yodo alcohol & RR (95\% IC) & Valor p \\
\hline Infección herida & $4,0 \%$ & $7,3 \%$ & $0,55(0,34-0,90)$ & 0,02 \\
Infección superficial & $3,0 \%$ & $4,9 \%$ & $0,61(0,34-1,10)$ & 0,10 \\
Infección profunda & $1,0 \%$ & $2,4 \%$ & $0,43(0,17-1,11)$ & 0,07 \\
\hline
\end{tabular}

\section{B. El estudio (1)}

Diseño: Estudio clínico randomizado, controlado, ciego, realizado en el periodo comprendido entre septiembre del 2011 y junio del 2015 en el centro médico de la Universidad de Washington, Saint Luis, Estados Unidos. Pacientes: 572 mujeres fueron randomizadas al grupo clorhexidina y 575 al grupo yodo. Intervención: Preparación preoperatoria de la piel con una solución de clorhexidinaalcohol (2\% clorhexidina - $70 \%$ isopropil alcohol). Comparación: Preparación preoperatoria de la piel con una solución de yodo-alcohol (8,3\% povidona iodada - $72,5 \%$ isopropil alcohol). Resultado primario: Infección superficial o profunda del sitio quirúrgico, dentro de 30 días siguientes a la operación cesárea. Resultados secundarios: Longitud de la estadía hospitalaria, visitas ambulatorias al médico, reingreso al hospital por complicaciones infecciosas, endometritis, cultivo positivo de la herida, irritación de la piel y reacciones alérgicas. Resultados: Como se observa en la Tabla I, la infección de la herida operatoria fue significativamente menor en el grupo asignado a clorhexidina, comparado con el grupo tratado con povidona yodada. Entre los resultados secundarios, la única diferencia significativa fue una menor incidencia de visitas al médico por complicaciones de la herida, en el grupo tratado con clorhexidina.

\section{Análisis Crítico}

Validez interna: Estudio con descripción apropiada de los criterios de inclusión y exclusión, randomizado, con ocultamiento de la secuencia de randomización, similitud de pacientes en ambos grupos, con seguimiento completo y análisis por intención de tratar. El tamaño muestral fue calculado para detectar una reducción de $50 \%$ en el riesgo de infección de la herida en el grupo tratado con clorhexidina, y se completó este número. El estudio no fue diseñado para mostrar diferencias significativas de los resultados secundarios. Los pacientes y los tratantes no fueron ciegos a la intervención, pero si lo fue el investigador principal quien fue el asignador de resultados, de modo que es muy poco probable que esta falta de ciego sea una fuente de sesgo. La infección de la herida operatoria fue definida por criterios específicos recomendados por el CDC. Validez externa: El tipo de pacientes incluidos y la incidencia de infección de herida operatoria son similares a la mayoría de las poblaciones mundiales, incluyendo la nuestra. Los agentes utilizados son similares a los usados en nuestro medio. Conclusión: Estudio con bajo riesgo de sesgo, de modo que sus resultados deben ser considerados como válidos y son suficientes como para asegurar un cambio en el uso habitual. En base a los resultados presentados, recomendamos el uso de una mezcla de clorhexidina con alcohol como el antiséptico de elección en la preparación preoperatoria de la piel antes de la operación cesárea. La mezcla de povidona yodada con alcohol solo debe ser reservada para mujeres con alergia a la clorhexidina.

\section{REFERENCIAS}

1. Tuuli MG, Liu J, Stout MJ, Martin S, Cahill AG, Odibo AO, Colditz GA, Macones GA. A Randomized Trial Comparing Skin Antiseptic Agents at Cesarean Delivery. N Engl J Med 2016;18;374(7):647-55.

2. Ministerio de Salud Chile. Panorama de Salud 2013, Informe OCDE sobre Chile y comparación con países miembros. Disponible en: http://www. cooperativa.cl/noticias/site/artic/20131122/asocfile/20131122092222/informe.pdf.

3. Conroy K, Koenig AF, Yu YH, Courtney A, Lee HJ, Norwitz ER. Infectious morbidity after cesarean delivery: 10 strategies to reduce risk. Rev Obstet Gynecol 2012;5(2):69-77. 